\title{
Early cervical cancer recurred with uterine fundus metastasis after laparoscopic fertility-sparing surgery: A case report
}

\section{Yuanming Shen (D 5312010@zju.edu.cn )}

Zhejiang University School of Medicine Women's Hospital

\section{Xiaoyun Wan}

Zhejiang University School of Medicine Women's Hospital

Jihong Liu

Sun Yat-sen University Cancer Center

\section{Xing Xie}

Zhejiang University School of Medicine Women's Hospital

\section{Case report}

Keywords: Cervical squamous cell carcinoma, Fertility-sparing surgery, Radical trachelectomy

Posted Date: June 5th, 2020

DOI: https://doi.org/10.21203/rs.3.rs-32297/v1

License: (c) (i) This work is licensed under a Creative Commons Attribution 4.0 International License. Read Full License 


\section{Abstract \\ Purpose}

Recently, the safety of minimally invasive radical hysterectomy for cervical cancer caused widespread controversy.The value of minimally invasive fertility-sparing surgery for early stage cervical cancer is still lacking evidence of randomized controlled trial (RCT).

\section{Methods}

Here we present a case of stage IB3 (FIGO2018) cervical carcinoma undergone neoadjuvant chemotherapy plus laparoscopic radical trachelectomy (A clinical trial NCT02624531) because of strongly desiring to preserve the fertility. Nine months after the initial treatment, the patient was presented with an uterine fundus mass and elevated serum squamous cell antigen (SCC Ag) and OC125 antigen (CA125).

\section{Results}

Cervical cancer uterine fundus involvement may occur with advanced tumor stage. Early stage cervical cancer relapsed with an uterine fundus metastasis after laparoscopic radical trachelectomy is exceptionally rare. The patient was treated and cured by a radical hysterectomy.Post-operative pathology confirmed an isolated implanted squamous cell carcinoma in uterine fundus and no previous surgerical margins and endometria were involved.

\section{Conclusions}

This firstly reported case indicates that the safety of laparoscopic radical trachelectomy is still uncertain for advanced stage cervical cancer even if neoadjuvant and post-surgery chemotherapy is given, and also suggests that it is needed to discuss carefully the benefit and risk of such a strategy with the patient when the fertility preservation is considered.

\section{Introduction}

Cervical cancer is still common malignancy among women worldwide. Following cervical screening popularization, up to $40 \%$ of early stage tumors are diagnosed in reproductive age, and some of them desire childbearing [1]. Fertility-sparing surgery has been an indispensible option for young, childbearing desired patients with early stage cervical cancer. Up to date, various procedures of fertility-sparing surgery for early stage cervical cancer have been reported, such as cone resection, simple trachelectomy, vaginal radical trachelectomy (VRT, Dargent's procedure), abdominal radical trachelectomy (ART), laparoscopic or robotic radical trachelectomy, and fertility-sparing surgery following neoadjuvant chemotherapy [2]. 
From a pragmatic point of view, the choice among them depends on four major factors: the prognostic factors of the tumor [size, stage, and lymph-vascular space invasion (LVSI) status]; the skill of the gynecological oncologist and the wider team treating the patient; morbidity; and fertility outcomes for the different fertility-sparing procedures[2].

Laparoscopic radical trachelectomy was first described in 2002 [2]. According to a literature review, 260 patients reported in 19 series were underwent full laparoscopic radical trachelectomy, and 138 patients reported in 10 series were underwent robotic radical trachelectomy till 2016 [2]. Laparoscopic surgery has gained worldwide acceptance as a surgical treatment for small early stage cervical cancers and presents better perioperative results compared with laparotomic routes, including less blood loss and a shorter hospital stay [2-4]. But the oncological outcomes of laparoscopic fertility-sparing surgery were still uncertain, especially in the larger tumours which reduced tumour size to eligible for radical trachelectomy after neoadjuvant chemotherapy, because reported data were limited.

Previous retrospective studies revealed that the safety of laparoscopic radical hysterectomy was equal to that of laparotomic radical hysterectomy [2].But recently, both prospective and retrospective studies in the New England Journal of Medicine showed that the minimally invasive radical hysterectomy was associated with higher recurrence rates and worse overall survival (OS), compared to laparotomic radical hysterectomy in women with stage IA2 or IB1 (Federation of Gynecology and Obstetrics, FIGO 2012) cervical cancer [5-6]. There still has been no study to compare the safety between minimally invasive and laparotomic radical trachelectomy though such a minimally invasive approach is commonly used in clinic.

Here, we reported the first case who presented uterine fundus implantation of cervical squamous cell carcinoma, after laparoscopic radical trachelectomy. This special case awakes us to rethink the safety of this fertility-sparing procedure for locally advanced cervical cancer patients.

\section{Case Presentation}

A 28-year-old nulligravid woman, who was diagnosed as locally advanced squamous cell cervical carcinoma stage IB3 (FIGO 2018) and undergone neoadjuvant chemotherapy followed by laparoscopic radical trachelectomy with pelvic lymphadenectomy 15 months ago, presented with an uterine fundus mass and elevated serum squamous cell antigen (SCC Ag) and OC125 antigen (CA125) .

Fifteen months ago, the patient was admitted to Department of Gynecologic Oncology, Women's Hospital, Zhejiang University School of Medicine, for intermittent and persistent vaginal spotting. Magnetic Resonance Imaging (MRI) revealed a $4.5 \mathrm{~cm} \times 3.8 \mathrm{~cm} \times 3.0 \mathrm{~cm}$ cervical mass involving cervix stromal invasion, no parametrial and vaginal infiltration, no cervical canal involvement and no lymphadenopathy. Biopsy confirmed well differentiated squamous cell carcinoma of the cervix. After informed-consent, she participated in a clinical trial (NCT02624531) because of strongly desiring to preserve the fertility. She received neoadjuvant chemotherapy with combined paclitaxel $175 \mathrm{mg} / \mathrm{m}^{2}$ and cisplatin $75 \mathrm{mg} / \mathrm{m}^{2}$ at 
three-week intervals. After two cycles, both MRI and colposcopy demonstrated partial response, with a reduction in tumor size to $1.8 \mathrm{~cm} \times 1.0 \mathrm{~cm} \times 2.1 \mathrm{~cm}$ (Fig. $1 \mathrm{a}-\mathrm{d})$, then she underwent laparoscopic radical trachelectomy with pelvic lymphadenectomy. Post-surgical pathology examination revealed well differentiated invasive squamous cell carcinoma, with some histopathological changes such as eosinophilic cytoplasm, vaculation, and enlarged nuclei (Fig.1e-h). No positive margins, nodal spread, and lymphovascular space involvement were found. After surgery, the patient underwent further four cycles of chemotherapy using the same regimen as that before surgery. However, nine months after treatment ended, the patient was found elevated serum SCC Ag of $2.1 \mathrm{ng} / \mathrm{mL}$ (reference, $<1.5 \mathrm{ng} / \mathrm{mL}$ ) and CA125 of $106 \mathrm{u} / \mathrm{mL}$ (reference, $<35 \mathrm{u} / \mathrm{mL}$ ) again. Both human papillomavirus testing and cytology were negative. Colposcopy showed that cervix and vagina were negative. Two weeks later, serum SCC Ag and CA125 were further elevated to $3.8 \mathrm{ng} / \mathrm{mL}$ and $223 \mathrm{u} / \mathrm{mL}$, respectively. Pelvic transvaginal untrasound and MRI showed a solitary $4.0 \mathrm{~cm} \times 3.5 \mathrm{~cm} \times 4.1 \mathrm{~cm}$ homogeneous solid mass at the uterine fundus (Fig.2a). Chest and abdomen computed tomography (CT) scans did not show the metastases out of the uterus. The recurrence of cervical carcinoma was diagnosed. The patient underwent laparoscopy. A $4.0 \mathrm{~cm} \times 4.0$ $\mathrm{cm}$ large, well-demarcated, nonencapsulated tumor with white color was found in uterine fundus (Fig.2b), then type $\mathrm{C}$ radical hysterectomy and bilateral adnexectomy were performed. Final histopathologic examination showed squamous cell carcinoma in uterine fundus (Figure2c-d), bilateral fallopian tube and ovarian were tumor-free. At the time of the manuscript submitting, the patient was alive with disease-free.

\section{Discussion}

The main spread pattern of cervical squamous cell carcinoma is loco-regional invasion, including laterally into the parametrium and distally into the upper vagina, but less often anterior-posteriorly into the bladder or rectum [7]. Spreading to regional pelvic nodes is common, but hematogenous metastases to lungs, liver, brain, etc. is unusual until late in advanced stages of cervical cancer with high grade tumors [9]. Another potential anatomical direction of direct extension in cervical cancer is into the uterine corpus. Retrospective study showed that the uterine corpus tumor invasion is rare in early-stage cervical cancer, especially in squamous histology [7]. A previous study identified 837 (4.9\%) cases of uterine corpus invasion in 17074 surgically-treated cervical cancer and found that uterine corpus invasion was independently associated with older age $(>50)$, non squamous histology, high-grade tumors, large tumor size $(>4 \mathrm{~cm})$, and nodal metastasis [8]. Non-squamous histology including adenocarcinoma is an independent risk factor for uterine corpus tumor invasion compared to squamous type [8]. The most likely causality of this association is the anatomical proximity to the uterine corpus of the endocervical gland origination of cervical adenocarcinoma. However, cervical squamous carcinoma usually develops at the exocervix and infrequently involves the uterine corpus. Uterine corpus tumor invasion has not been incorporated into the cervical cancer staging system, up to date [8]. Here we reported a case of metastatic uterine corpus cancer that was secondary to a stage IB3 squamous cell cervical cancer. This patient underwent neoadjuvant chemotherapy and laparoscopic radical trachelectomy. She had no surgicalpathological risk factors, but presented a uterine fundus metastasis in a short interval from primary surgery. Pathologic examination confirmed that metastatic squamous carcinoma of uterine fundus was 
isolated and did not reach the margin of previous surgery, and endometrium was also not involved, suggesting a very low possibility that the lesion of uterine fundus is a direct extension from original cancer of the cervix. One explanation may be that tumor cells in uterine fundus was carried into by uterine manipulator, which is frequently used for retraction and visualization, during minimally invasive surgery.

Radical trachelectomy is typically only for stage IB1 patients with tumor $2 \mathrm{~cm}$ or less in diameter, negative nodes, and non-aggressive histological subtypes [9]. However, some surgeons proposed that neoadjuvant chemotherapy could be used for patients with locally advanced stage to reduce tumor size so that fertility was preserved [9]. Platinum-based neoadjuvant chemotherapy has been shown to improve local control (reduce tumour size and parametrial infiltration) and offer a better control of micrometastases in distant sites as well as in regional lymph nodes [10]. In a previous report, totally 25 cervical cancer staging IB3 (FIGO2018) women underwent neoadjuvant chemotherapy plus conservative surgery, including 10 simple vaginal trachelectomy, 10 vaginal radical trachelectomy, and 5 abdominal radical trachelectomy, among them only $2(8 \%)$ recurred [2]. Considering the limitations of retrospective studies, we are conducting a prospective clinical trial (NCT02624531) about the application of fertility-sparing therapy for patients with cervical cancer staging IB1-IIA2 (FIGO2012). According to the protocol, the patient with stage IB3 (FIGO2018) squamous cell cervical carcinoma is assigned to receive two or three cycles of neoadjuvant chemotherapy followed by radical trachelectomy and further three to four cycles of adjuvant chemotherapy after surgery. The patient we reported presented partial remission (PR) to neoadjuvant chemotherapy, which meant that tumor was still existed in the cervix, though the size was reduced, after neoadjuvant chemotherapy. Accordingly, we have reason to speculate that the tumor cells probably moved from cervix and implanted into uterine fundus by uterine manipulator during surgery. Thus, our special case also suggests that it should be considered to avoid the use of uterine manipulator during minimally invasive fertility-sparing surgery or perform abdominal radical trachelectomy instead.

In summary, we reported a special case of uterine fundus implanted cervical squamous cell carcinoma. The patient was diagnosed with stage IB3 (FIGO 2018) and underwent neoadjuvant chemotherapy plus laparoscopic radical trachelectomy. Pathologic diagnosis was well-differentiated squamous cell carcinoma without pathologic risk factors. The patient suffered from metastatic cancer in uterine fundus later and underwent radical surgery finally. This case with special metastatic manner indicates that the safety of laparoscopic radical trachelectomy is still uncertain for advanced stage cervical cancer even if neoadjuvant and post-surgery chemotherapy is given, and also suggests that it is needed to discuss carefully the benefit and risk of such a strategy with the patient when the fertility preservation is considered.

\section{Abbreviations}

SCC Ag

erum squamous cell antigen

CA125

OC125 antigen 
FIGO

Federation of Gynecology and Obstetrics

VRT

vaginal radical trachelectomy

ART

abdominal radical trachelectomy

LVSI

lymph-vascular space invasion

OS

overall survival

MRI

Magnetic Resonance Imaging

CT

computed tomography

\section{Declarations}

Ethics approval and consent to participate: This research conformed to the provisions of the Declaration of Helsinki. The patient was informed and provided her written informed consent. This study was approved by the ethics committee of Women's hospital Zhejiang University

Consent for publication: The patient was informed and provided her written informed consent.

Availability of data and materials: The datasets used and analysed during the current study are available from the corresponding author on reasonable request

Competing interests: we certify that no actual or potential conflict of interest in relation to this article exists. We also certify that non-financial competing interests include political, personal, religious, ideological, academic, and intellectual competing interests.

Funding: Natural Science Foundation of Zhejiang province ChinaLY19H160043. The role the funding is to supported the clinical trial (NCT02624531).

Authors' contributions: Yuanming Shen and Xie Xing made substantial contributions to the conception.Jihong Liu and Xie Xing design of the work; Yuanming Shen and Xiaoyun Wan interpretation of data; Yuanming Shen have drafted the work; Jihong Liu and Xie Xing substantively revised it.

Acknowledgements: Thank you for DongXiao Hu who provided only general support.

\section{References}


1. Bray F, Ferlay J, Soerjomataram I, Siegel RL, Torre LA, Jemal A.Global cancer statistics 2018: GLOBOCAN estimates of incidence and mortality worldwide for 36 cancers in 185 countries. CA Cancer J Clin.2018; 68(6):394-424.

2. Bentivegna E, Gouy S, Maulard A.Oncological outcomes after fertility-sparing surgery for cervical cancer: a systematic review.Lancet Oncol.2016;17(6):e240-e253

3. Diver E, Hinchcliff E, Gockley A, Melamed A, Contrino L, Feldman S, Growdon W. Minimally invasive radical hysterectomy for cervical cancer is associated with reduced morbidity and similar survival outcomes compared with IJ Minim Invasive Gynecol.2017; 24 (3): 402-406.

4. Rizou N, Moris D, Pikoulis E, Dimitrokallis N, Mpaili E, Felekouras E,Papalampros A .Minimally invasive lymphadenectomy in uterine cervical cancer a systematic review.Anticancer Res. 2017;37(1): $335-342$.

5. Ramirez PT, Frumovitz M, Pareja R, Lopez A, Vieira M, Ribeiro R, Buda A, Yan X, Shuzhong Y, Chetty N, Isla D, Tamura M, Zhu T, Robledo KP, Gebski V, Asher R, Behan V, Nicklin JL, Coleman RL, Obermair A Minimally invasive versus abdominal radical hysterectomy for cervical cancer.N Engl $\mathrm{J}$ Med.2018;379(20):1895-1904.

6. Melamed A, Margul DJ, Chen L, Keating NL, Del Carmen MG, Yang J, Seagle BL, Alexander A, Barber EL, Rice LW, Wright JD, Kocherginsky M, Shahabi S,Rauh-Hain JA.Survival after minimally invasive radical hysterectomy for early-stage cervical cancer.N Engl J Med.2018;379(20):1905-1914.

7. Barakat RR, Markman M, Randall ME, editors . Principal and practice of gynecologic oncology. 5th ed. Philadephia: Lippincott Williams \& Wilkins;New York,2009;pp623-

8. Matsuo K, Machida H, Blake EA, Takiuchi T, Mikami M, Roman LD . Significance of uterine corpus tumor invasion in early-stage cervical cancer. Eur J Surg Oncol.(2017);43(4):725-734.

9. Marchiole P, Tigaud JD, Costantini S, Mammoliti S, Buenerd A, Moran E, Mathevet P. Neoadjuvant chemotherapy and vaginal radical trachelectomy for fertility-sparing treatment in women aff ected by cervical cancer (FIGO stage IB-IIA1).Gynecol Oncol.2011;122: 484-490.

10. Robova H, Halaska MJ, Pluta M, Skapa P, Matecha J, Lisy J, Rob L.Oncological and pregnancy outcomes after high-dose density neoadjuvant chemotherapy and fertility-sparing surgery in cervical cancer.Gynecol Oncol.2014;135: 213-2

\section{Figures}



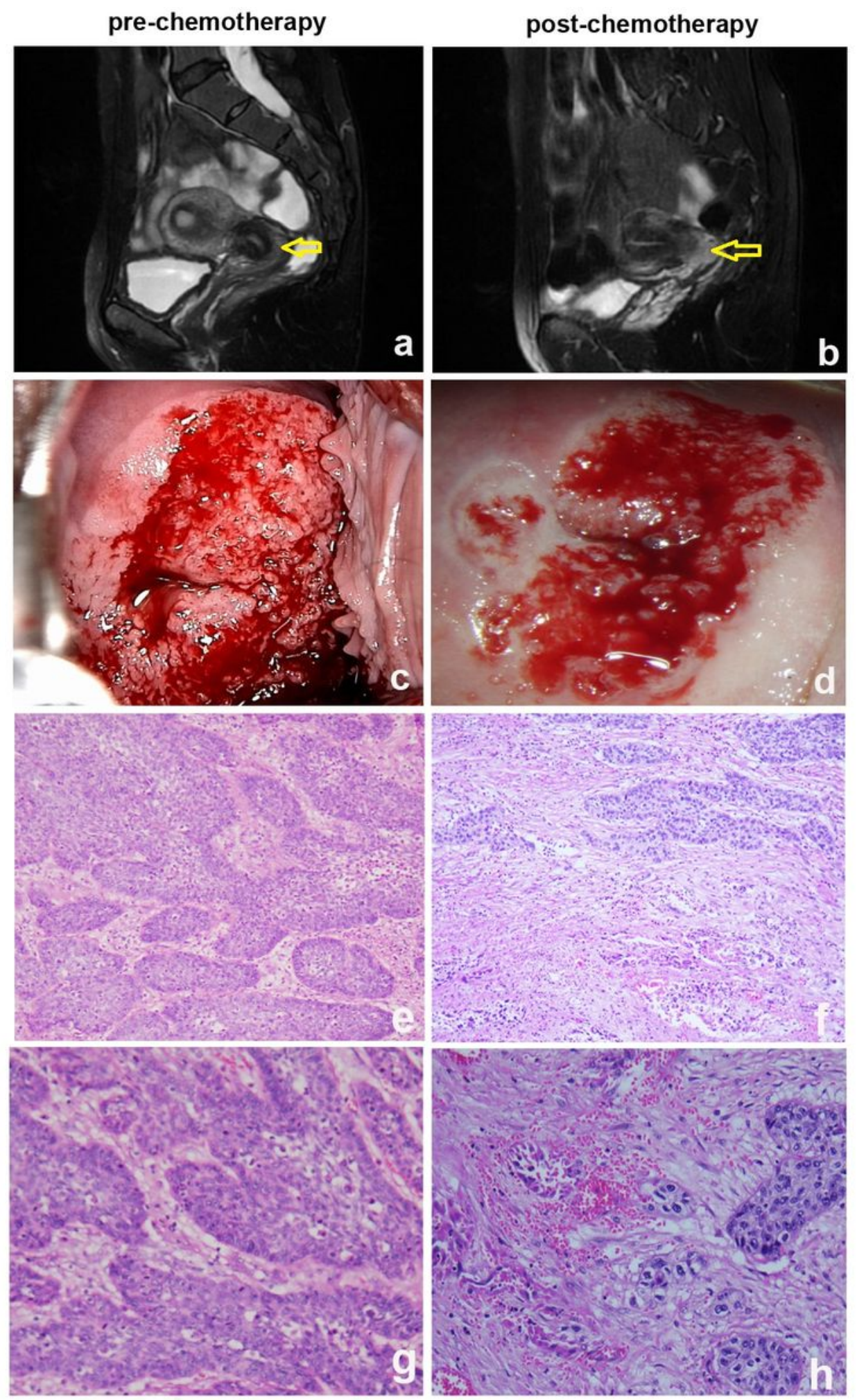

\section{Figure 1}

The cervical mass in MRI (arrow) and colposcopy pre- $(a, c)$ and post-chemotherapy $(b, d)$. Hematoxylin and eosin stain (20x magnification) demonstrating well differentiated invasive squamous cell carcinoma $(e, g)$. Post-surgical pathology indicated the effectiveness of chemotherapy characterized by the presence of eosinophilic cytoplasm, vacuolation, and enlarged nuclei $(f, h)$. 


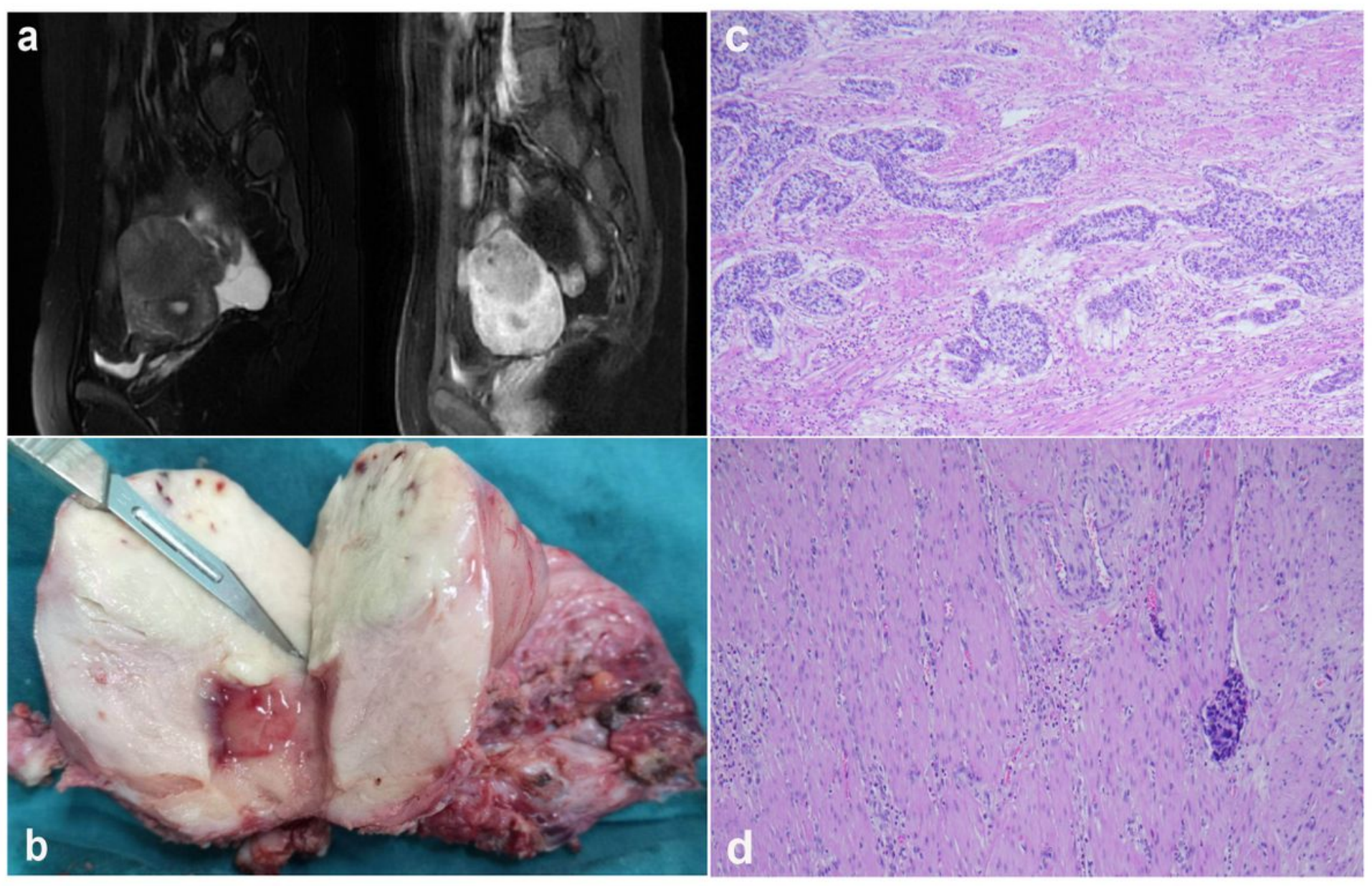

\section{Figure 2}

A MRI scan (a) of the uterine and pelvis. An isolated well-demarcated, nonencapsulated tumor in uterine fundus (b). An intramuscular uterine fundus squamous cell carcinoma with vascular infiltration (H\&E stain, 20x magnification) $(c, d)$. 